\title{
Patient-Activated Three-Dimensional Multifrequency Magnetic Resonance Elastography for High-Resolution Mechanical Imaging of the Liver and Spleen
}

\section{Patienten-aktivierte dreidimensionale Multifrequenz-Magnet- resonanzelastografie zur hochauflösenden mechanischen Bildgebung der Leber und Milz}

Authors

Affiliations
J. Guo ${ }^{1}$, S. Hirsch', K. J. Streitberger ${ }^{1}$, C. Kamphues², P. Asbach', J. Braun ${ }^{3}$, I. Sack ${ }^{1}$

Department of Radiology; Charité - Universitätsmedizin Berlin; Campus Charité Mitte; Berlin; Germany

Department of General, Visceral and Transplantation Surgery; Charité - Universitätsmedizin Berlin; Campus Virchow Clinic; Berlin; Germany

Institute of Medical Informatics; Charité - Universitätsmedizin Berlin; Campus Benjamin Franklin; Berlin; Germany
Key words

- magnetic resonance elastography

- MR imaging

- abdomen

- spleen

- liver received 15.3.2013 accepted 25.7.2013

\section{Bibliography}

DOI http://dx.doi.org/

$10.1055 / \mathrm{s}-0033-1350510$

Published online: 2.9.2013

Fortschr Röntgenstr 2014; 186 : 260-266 @ Georg Thieme Verlag KG Stuttgart · New York . ISSN 1438-9029

\section{Correspondence Ingolf Sack}

Institut für Radiologie, Charité Universitätsmedizin Berlin Charitéplatz 1

10117 Berlin

Germany

Tel.: ++ 49/0 30/450539058

Fax: ++49/030/450539988

ingolf.sack@charite.de

\section{Zusammenfassung}

$\nabla$

Ziel: Entwicklung und Demonstration einer neuen dreidimensionalen Mehrfrequenz-Magnetresonanzelastografie-Mehode (3-D-MMRE) zur hochauflösenden mechanischen in vivo Bildgebung der Leber und Milz.

Material und Methoden: Zehn gesunde Freiwillige wurden mittels abdominaler single-shot 3-DMMRE unter Anwendung eines neuartigen Patienten-aktivierten Atemtrigger-Systems untersucht. Zeitaufgelöste Wellenfelder in zehn zusammenhängende Schichten mit 2,5 mm kubischer Pixel-Auflösung, 3 Wellenkomponenten, 8 Zeitschritten, 2 Mittelungen und 7 Frequenzen zwischen 30 und $60 \mathrm{~Hz}$ wurden innerhalb von 15 min aufgenommen und mittels Mehrfrequenzdual-elasto-visco (MDEV) Inversion analysiert.

Ergebnisse: Karten der zwei unabhängigen mechanischer Kenngrößen, Betrag und Phase des komplexen Moduls, wurden in hoher räumlicher Auflösung erzielt. Details der mechanischen Struktur der Leber und der Milz sind gut erkennbar. Die Leber erscheint mit gemittelten Elastizitätswerte von $1,27 \pm 0,17 \mathrm{kPa}$ wesentlich weicher als die Milz, für die eine Elastizität von 2,01 \pm 0,69 kPa gefunden wurde.

Schlussfolgerung: Patienten-aktivierte 3-D-MMRE kombiniert mit MDEV-Inversion stellt die erste Modalität zur hochauflösenden mechanischen Bildgebung von Leber und Milz dar und verspricht damit genauere MRE-Untersuchungen als bisherige Systeme.

\section{Abstract \\ $\nabla$}

Purpose: To introduce a novel in-vivo three-dimensional multifrequency magnetic resonance elastography (3D-MMRE) method for high-resolution mechanical characterization of the liver and spleen.

Materials and Methods: Ten healthy volunteers were examined by abdominal single-shot 3DMMRE using a novel patient-activated trigger system with respiratory control. 10 contiguous slices with $2.5 \mathrm{~mm}$ cubic voxel resolution, 3 wave components, 8 time steps, and 2 averages were acquired at 7 mechanical excitation frequencies from 30 to $60 \mathrm{~Hz}$. The total imaging time was approximately $15 \mathrm{~min}$. For postprocessing, multifrequency dual elasto-visco (MDEV) inversion was used to calculate high-resolution mechanical parameter maps of the abdomen including the liver and spleen.

Results: Two parameters maps were generated from each image slice to capture the magnitude and the phase angle of the complex shear modulus. Both parameters depicted the mechanical structures of the abdomen with unprecedented high spatial resolution. Spatially averaged group mean values of the liver and spleen are $1.27 \pm 0.17$ $\mathrm{kPa}$ and $2.01 \pm 0.69 \mathrm{kPa}$, indicating a significantly higher asymptomatic stiffness of the spleen compared to the liver.

Conclusion: Patient-activated respiratory-gated 3D-MMRE combined with MDEV inversion provides highly resolved mechanical maps of the liver and spleen that are superior to previous elastograms measured by abdominal MRE.

Citation Format:

- Guo J, Hirsch S, Streitberger KJ etal. PatientActivated Three-Dimensional Multifrequency Magnetic Resonance Elastography for High-Resolution Mechanical Imaging of the Liver and Spleen. Fortschr Röntgenstr 2014; 186: 260-266 


\section{Introduction}

\section{$\nabla$}

The noninvasive mechanical examination of the liver provides a well-established marker of hepatic fibrosis [1 -4]. Techniques of medical ultrasound and magnetic resonance imaging (MRI) have been developed for the measurement of the mechanical response of the liver to mechanical stress [5]. Most of these modalities, subsumed under the term elastography, use static compression [6], externally induced shear waves $[7,8]$ or focused ultrasound pulses [9] to produce a controlled elastic deformation of the tissue.

MRI elastography (MRE) relies on time-harmonic shear waves [10] transmitted into the body from the surface, where they are generated by on-site vibrating coils or by passive transducers attached to a loudspeaker or to a piezoelectric driver [11 - 14]. To date, MRE is the only modality capable of measuring vector fields of shear waves in soft tissue with isotropic image resolution, which promotes a stable numerical solution of the inverse problem in elastography and therewith ultimately determines the diagnostic precision of elastography [15]. For this reason, the accuracy of MRE potentially outperforms that of other modalities with anisotropic motion estimation.

However, MRE still suffers from uncertainty regions in elastograms, which have to be masked in order to avoid biased stiffness estimates $[16,17]$. Such artifacts are mainly due to the strong impact of boundaries and tissue interfaces on the appearance of wave fields including non-evanescent waves, wave nodes, and amplitude modulations deviating from ideal wave models implied by the majority of inversion routines. To reduce such wave voids, multifrequency inversion was recently introduced, which adds wave fields of different drive frequencies to the solution of an overdetermined system of wave equations [18]. As a result, the accuracy of elastograms can be greatly improved, depending on the amount of information combined into a single inversion step [14]. In this approach, the major limiting factor for improving the quality of elastograms is the duration of image acquisition. For example, single-shot MRE requires repetitive scans for each image slice, wave component, time increment, drive frequency, and, if required, signal averages [19]. Abdominal applications are particularly challenging as they require additional respiratory gating.

Previous hepatic MRE studies were run in 3D at single drive frequency [20-22] or at multiple frequencies in $2 \mathrm{D}[13,23]$ with voxel volumes of $64 \mathrm{~mm}^{3}$ or larger. Recently, we introduced 3D multifrequency MRE (3D-MMRE) with four vibration frequencies and an image resolution of $2.7 \times 2.7 \times 5 \mathrm{~mm}^{3}$ for better resolved and less biased MRE of the liver and spleen [14].

In order to further improve the resolution of abdominal MRE, the quality of the wave images has to be increased (for improved detail resolution of anatomical structures) and more vibration frequencies should be applied (in order to stabilize the inversion by independent wave fields) [18]. To achieve this in a clinically feasible protocol, this study introduces a patient-activated respiratory gating system for abdominal 3D-MMRE. Using this novel gating method, the patient can actively control the time intervals for image acquisition and breathing, which effectively reduces the total examination time and enables us to acquire dynamic wave field data in 10 slices at a resolution of $2.5 \times 2.5 \times 2.5 \mathrm{~mm}^{3}$ and at 7 vibration frequencies within a clinically acceptable time frame.

We use this enhanced elastodynamic information to produce mechanical parameter maps which display details of the abdomen including the liver and the spleen with fewer artifacts than previous methods. This methodological improvement is intended to provide the methodological background for a precise mechanical characterization of abdominal tissue in different clinical applications such as the assessment of lesions, staging of heterogeneous hepatic fibrosis, or detection of a vascular pressure imbalance, which may influence the elasticity ratio between the liver and spleen [24].

\section{Methods \\ $\nabla$}

The study was approved by the institutional review board. Ten healthy volunteers ( 2 females, mean age, $34.5 \pm 8.7$ years) were investigated using a clinical protocol including T1-, T2-, and proton-density-weighted pulse sequences. Furthermore, 3D-MMRE was applied as described in the following.

\section{D-MMRE}

A custom-designed nonmagnetic driver based on piezoelectric ceramics as proposed by [25] was mounted at the end of the patient table ( $\bullet$ Fig. 1). The vibrations were transferred from the tip of the lever to the patient by a carbon fiber rod connected at its distal end to a rubber mat. The transducer mat was moderately pressed onto the body surface in the position just below the right costal arch using Velcro strips attached to the patient table [14]. All experiments were conducted on a 1.5-T MRI scanner (Magnetom Sonata; Siemens Erlangen, Germany) equipped with a 12channel phased-array surface coil. A 2 D single-shot spin-echo echo-planar imaging (EPI) sequence with a trapezoidal flowcompensated motion-encoding gradient (MEG), consecutively applied along all three axes of the scanner coordinate system, was used for rapid motion field acquisition. The motion was initiated by a trigger pulse from the scanner at least $100 \mathrm{~ms}$ before the start of the MEG to allow the waves to propagate into the tissue. Eight dynamics of a wave cycle were captured by delaying the trigger pulse in increments of $1 /(8 f)$ with $f$ being the vibration frequency. The vibration frequencies were $30,35,40,45,50,55$, and $60 \mathrm{~Hz}$. For each drive frequency, MEG direction, and wave dynamic, 10 adjacent transverse image slices with a resolution of $2.5 \times 2.5 \times 2.5 \mathrm{~mm}^{3}$ were recorded, yielding a total of 1680 images. For each image, the signal was averaged twice to improve the signal-to-noise ratio (SNR). Further imaging parameters were: repetition time (TR), $3790 \mathrm{~ms}$; echo time (TE), $55 \mathrm{~ms}$; field of view (FoV), $320 \times 260 \mathrm{~mm}^{2}$; matrix size, $128 \times 104$; parallel imaging with a GRAPPA of factor 2; MEG frequency, $50 \mathrm{~Hz}$ (implying fractional motion-encoding for all drive frequencies $\$ 50 \mathrm{~Hz}[26]$ and providing a feasible compromise between motion sensitivity and short TE); MEG amplitude, $30 \mathrm{mT} / \mathrm{m}$; net scan time (without respiratory gating) for each frequency, $1.5 \mathrm{~min}$. The total examination time (for 7 frequencies) was approximately $13 \mathrm{~min}$, with variations depending on the voluntarily adjusted breathing intervals.

\section{Patient-activated respiratory gating}

Each volunteer was asked to maintain a reproducible position of the chest and abdomen in expiration through the patient-activated 3D-MMRE acquisition. When activated, the trigger delayed the processing of wave image acquisition after accomplishing an entire TR-interval. Therefore, the halt of the sequence did not interfere with the k-space acquisition of single images, and the respiratory pauses could be introduced anytime during the acquisition of full wave images of different vibration phases or field components. 


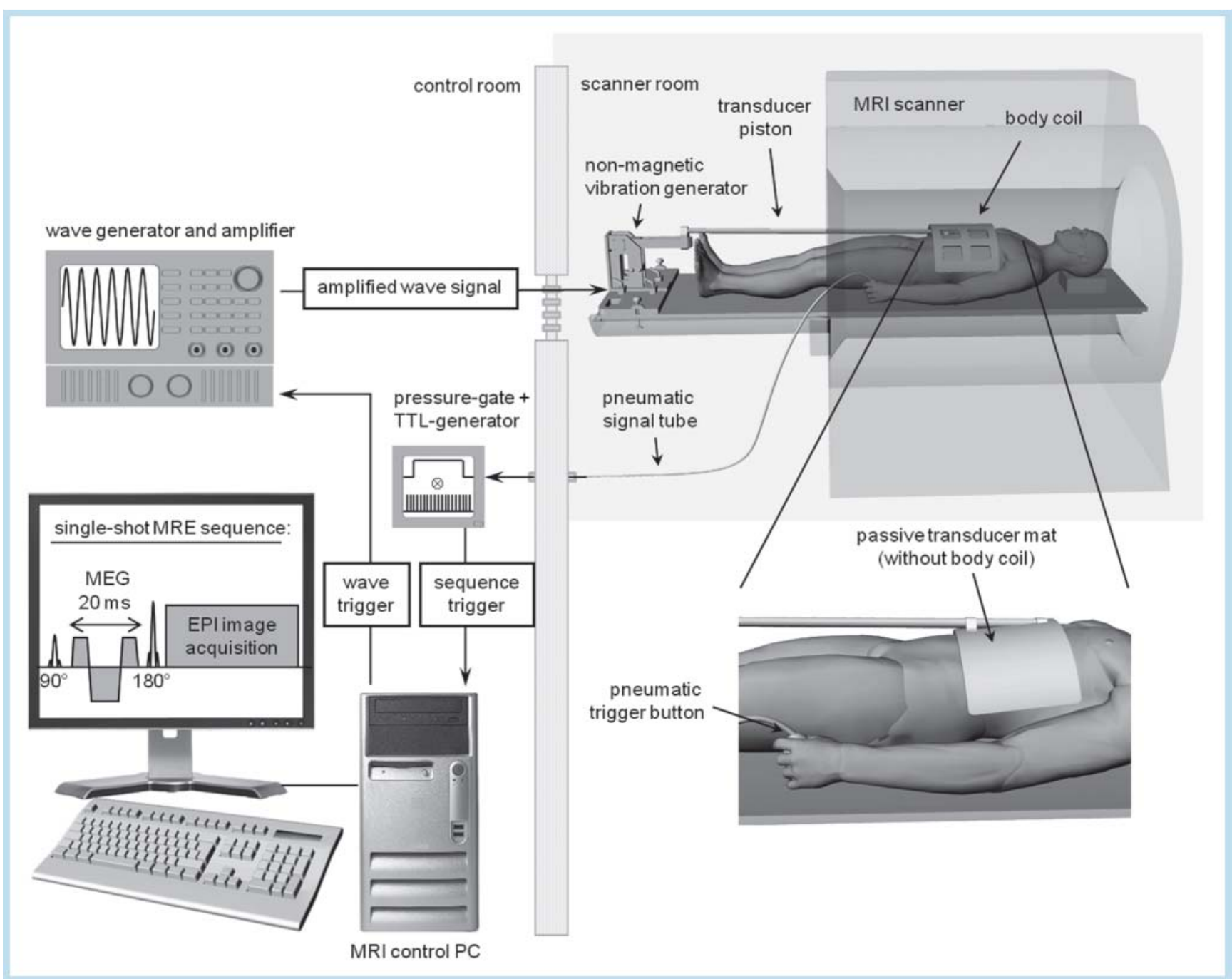

Fig. 1 Experimental setup of patient-activated 3D-MMRE. Details of the MRE sequence and the nonmagnetic vibration generator are given in [14]. The novel patient-activated respiratory gating system is described in the method section.

Abb. 1 Experimenteller Aufbau für patientenaktivierte 3-D-MMRE. Details über die MRE-Sequenz und den nichtmagnetischen Vibrationserzeuger sind in [14] publiziert. Der neuartige patientenaktivierte Trigger ist im Methodenteil beschrieben.

For activating the trigger, a pneumatic ball was connected to a pressure-sensitive button which produced a continuous $4.5-\mathrm{V}$ gating signal fed into a signal generator outside the scanner room. The signal generator produced a comb of equidistantly spaced rectangular TTL pulses with a frequency of $100 \mathrm{~Hz}$ and a $50 \%$ duty cycle. Thus, upon activation, the pneumatic ball shown in $\bullet$ Fig. 1 generated the TTL-comb-shaped signal for starting the externally triggered 3D-MMRE sequence. The signal pathway is schematically shown in $\bullet$ Fig. 1.

\section{Data processing}

In the first step of postprocessing, derivative-based unwrapping was performed. This method is a useful tool for treating phase wraps if the derivative of the displacement field is further considered (in our case when applying the curl-operator). The method was proposed several years ago in reference [27] and has been used since then as a standard procedure in MRE. First-order spatial derivatives of the $m$-th component of spin phase $\varphi_{m}$ were calculated using the following equation:

$$
\frac{\partial \varphi_{m}\left(r, \omega_{n}^{2}\right)}{\partial x_{k}}=-i \cdot \exp \left[-i \varphi_{m}\left(r, \omega_{n}\right)\right] \cdot \frac{\partial}{\partial x_{k}} \exp \left[i \varphi_{m}\left(r, \omega_{n}\right)\right]
$$

where $\varphi_{m}\left(\mathbf{r}, \omega_{n}\right)$ denotes the phase measured at n-th external angular drive frequencies and position $\mathbf{r} . \varphi_{m}$ is wrapped $\left(\varphi_{m} \in[0,2 \pi)\right)$, while its derivative is wrap-free and unbiased if the phase difference of two adjacent voxels does not exceed $\pi . \mathbf{r}$ denotes the position vector and the $n$-th angular drive frequency. $\partial \varphi_{m} \partial x_{k}$ was scaled to $\partial u_{m /} \partial x_{k}$ (i.e., spatial derivatives of the physical displacement field $\mathbf{u}$ ) by the factor given in equation (4b) of [28].

For reducing noise, numerical derivatives were calculated by 3D gradients according to Anderssen and Hegland [29] using a twopixel symmetric window in three dimensions [30]. The resulting strain components of the wave field (derivatives of the three Cartesian components of the displacement field) were used to calculate the curl field, as has been established in 3D MRE for suppressing volumetric strain [31]. The curl components $C_{1}, C_{2}$ and $C_{3}$ are defined as follows:

$C_{1}=\frac{\partial \mathrm{u}_{3}}{\partial \mathrm{x}_{2}}-\frac{\partial \mathrm{u}_{2}}{\partial \mathrm{x}_{3}} ; \mathrm{C}_{2}=\frac{\partial \mathrm{u}_{1}}{\partial \mathrm{x}_{3}}-\frac{\partial \mathrm{u}_{3}}{\partial \mathrm{x}_{1}} ; \mathrm{C}_{3}=\frac{\partial \mathrm{u}_{2}}{\partial \mathrm{x}_{1}}-\frac{\partial \mathrm{u}_{1}}{\partial \mathrm{x}_{2}}$. 

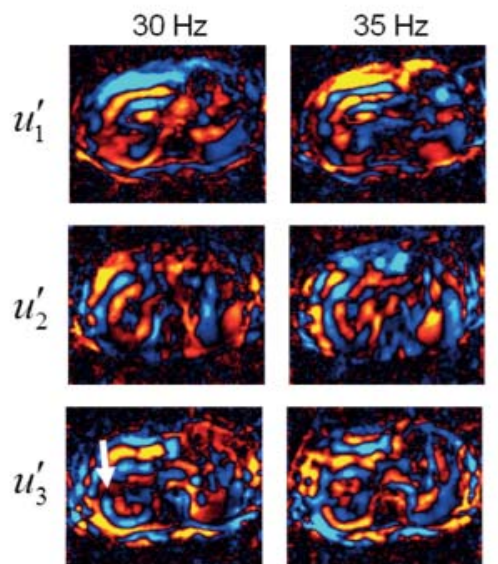
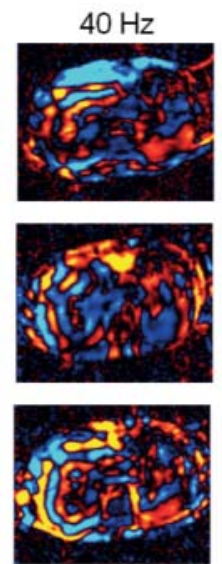
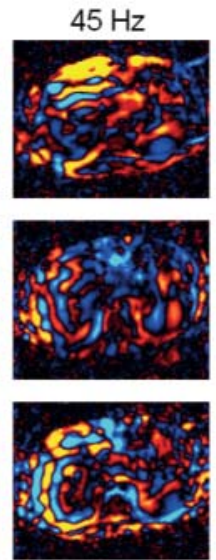
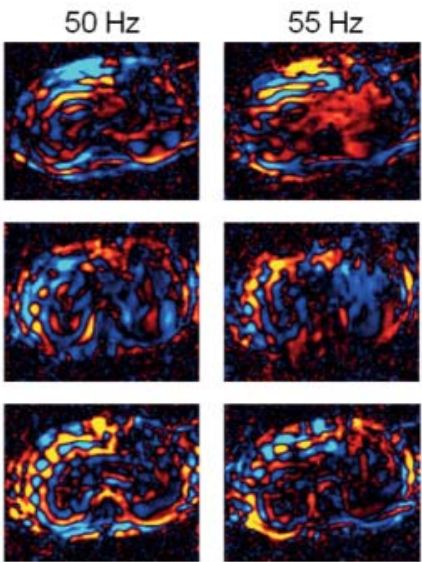
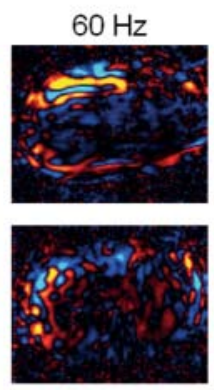

Fig. 2 Components of shear strain fields in a central slice of the liver of a volunteer at 7 vibration frequencies and 3 motion encoding directions. The white arrow at $30 \mathrm{~Hz}$ indicates wave nodes due to destructive interferences while the arrow at $60 \mathrm{~Hz}$ demarcates viscous damping.

Abb.2 Die Komponenten der Scherverzerrungsfelder in einer zentralen Schicht durch die Leber eines Freiwilligen bei 7 Vibrationsfrequenzen. Der weiße Pfeil markiert einen streubedingten Knoten des Feldes.

where $u_{m}(\mathbf{r}, t)$ is the $m$-th component of the wave field with $m=1$, 2 , and 3 referring to the imaging gradient directions of read-out, phase-encoding, and slice selection, respectively. The curl components of equation (2) were smoothed by a 3D Gaussian kernel with an edge length of 3 pixels and were Fourier-transformed along the time axis (eight samples over one vibration cycle). The resulting complex 3D wave images, corresponding to the first harmonic $n$-th vibration frequency $\left(\omega_{n}\right)$, were taken as components $u_{\mathrm{m}}^{*}\left(\mathbf{r}, \omega_{n}\right)$ of the wave field for multifrequency dual elastovisco (MDEV) parameter recovery as described in detail in [14]. In MDEV inversion, the complex-valued shear modulus $G^{*}$ is represented by its magnitude $\left|G^{*}\right|$ and phase angle $\varphi$. Both parameters are reconstructed separately using a modified Helmholtz inversion as briefly summarized in the appendix.

\section{Results}

\section{$\nabla$}

The new patient-activated gating mechanism was easily handled by all volunteers and was reported to support better reproduction of the breath-hold position than conventional breathing commands.

- Fig. 2 portrays a set of complex waves $u_{m}^{\prime}=\operatorname{Re}\left\{u_{m}^{*}\left(\mathbf{r}, \omega_{n}\right)\right\}$ obtained in a central slice of a volunteer at 7 vibration frequencies. The images were derived from the raw phase signal by gradient unwrapping (equation (1)), curl calculation (equation (2)), and temporal Fourier transformation. Application of the curl operator suppresses compression wave artifacts. The wave lengths decrease with increasing vibration frequency. It is well visible that the appearance of the wave patterns depends on the field component and frequency, which corroborates our approach of compensating for low wave amplitudes due to wave nodes (e.g., demarcated by the arrow in $u_{3}^{\prime}$ of $30 \mathrm{~Hz}$ ) or attenuation (e.g., at $60 \mathrm{~Hz}$ ) by combining all images in the inversions of equations (5) and (6).

- Fig. 3 highlights the appearance of MDEV parameter maps in a transverse slice of five volunteers. We arbitrarily chose 5 volunteers including the ones with the smallest and largest liver in or- der to cover a wide range of physiological variations in hepatic morphology. Other elastograms acquired in this study appear similar with regard to anatomical features and resolution.

In contrast to the classic display of elastograms in MRE $[12,13$, 32], the maps of mechanical parameters in 0 Fig. 3 are not masked for excluding noise or regions of unreliably reconstructed parameters. Instead, the full mechanical image can be evaluated including the liver and spleen. Visible heterogeneities are due to the blood vessel in the liver. The spleen appears to be stiffer and more heterogeneous than the liver as indicated by group mean values of $\left|G^{*}\right|=1.27 \pm 0.17 \mathrm{kPa}$ and $\left|G^{*}\right|=2.01 \pm 0.69 \mathrm{kPa}$ for hepatic and splenic stiffness and $\mathrm{SD}=0.35 \pm 0.08 \mathrm{kPa}$ and $\mathrm{SD}=0.69 \pm 0.34 \mathrm{kPa}$ for intrahepatic and intrasplenic heterogeneity ( $\odot$ Table 1$)$. Stiffness and heterogeneity are both significantly different with p-values of 0.0041 and 0.0066 , respectively. Similar statistically significant differences were found for $\varphi$, which was higher in the spleen $(0.607 \pm 0.152)$ than in the liver $(0.407 \pm 0.044, p=0.0008)$, again with a higher intrasplenic heterogeneity ( $S D=0.204 \pm 0.034$ [liver] vs. $0.282 \pm 0.076$ [spleen], $\mathrm{p}=0.0084$ ). Similar to previous studies $[24,33]$, we found a significant correlation between $\left|G^{*}\right|$ of the liver and spleen $(p=0.046)$, while $\varphi$ was not correlated in the two organs, as has been reported in [14].

\section{Discussion \\ $\nabla$}

High-resolution mechanical imaging remains a major goal of method development in elastography. Our study contributes to this development by introducing a new respiratory gating method that enables us to acquire more wave fields at different frequencies in the abdomen than was feasible with earlier techniques ( 7 frequencies, 2 averages compared to 4 frequencies, no average in Ref. [14]). This multidimensional information was combined into two maps of viscoelastic parameters which are related to the elasticity and viscosity of the tissue.

Our previous studies using MMRE in the liver [23, 34, 35] have shown that the liver is a highly viscous organ giving rise to signif- 


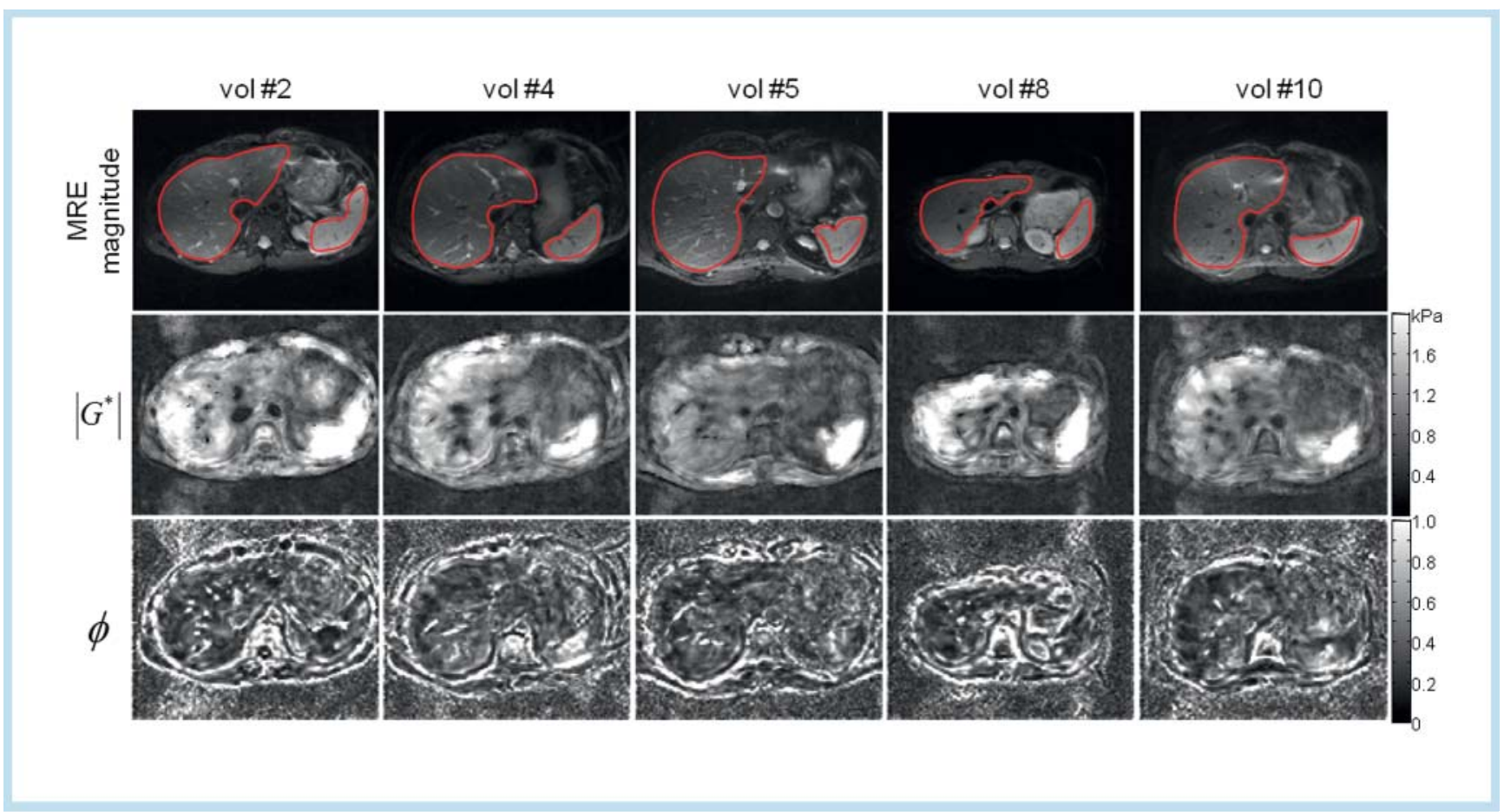

Fig. 3 3D-MMRE parameter maps in central slices of 5 volunteers. The volunteer numbers refer to Table 1 . Regions for spatial averaging of $\left|G^{*}\right|$ and $\varphi$ in the liver and the spleen are encircled by red lines.

Abb. 3 3-D-MMRE-Parameterkarten in den zentralen Schichten von 5 Freiwilligen. Die Nummerierung der Personen stimmt mit Tab. 1 überein. Die Mittelungsregionen für $\left|\mathrm{G}^{*}\right|$ und $\varphi$ in Leber und Milz sind in Rot eingezeichnet.

\begin{tabular}{|lllllllllll}
$\begin{array}{l}\text { vol. } \\
\#\end{array}$ & sex & $\begin{array}{l}\text { age } \\
\text { years }\end{array}$ & $\begin{array}{l}\text { liver } \\
\text { IG* } \\
\text { kPa }\end{array}$ & $\begin{array}{l}\text { liver } \\
\text { SD } \\
\mathbf{k P a}\end{array}$ & $\begin{array}{l}\text { spleen } \\
\mathbf{I}^{*} \mathbf{I} \\
\mathbf{k P a}\end{array}$ & $\begin{array}{l}\text { spleen } \\
\text { SD } \\
\mathbf{k P a}\end{array}$ & $\begin{array}{l}\text { liver } \\
\varphi\end{array}$ & $\begin{array}{l}\text { liver } \\
\text { SD }\end{array}$ & $\begin{array}{l}\text { spleen } \\
\varphi\end{array}$ & $\begin{array}{l}\text { spleen } \\
\text { SD }\end{array}$ \\
\hline 1 & $\mathrm{~m}$ & 43 & 1.23 & 0.25 & 2.60 & 1.12 & 0.374 & 0.172 & 0.587 & 0.225 \\
\hline 2 & $\mathrm{~m}$ & 27 & 1.41 & 0.43 & 2.84 & 0.76 & 0.397 & 0.229 & 0.414 & 0.245 \\
\hline 3 & $\mathrm{~m}$ & 27 & 1.47 & 0.39 & 1.52 & 0.58 & 0.450 & 0.291 & 0.753 & 0.260 \\
\hline 4 & $\mathrm{~m}$ & 30 & 1.30 & 0.41 & 1.99 & 0.51 & 0.411 & 0.198 & 0.816 & 0.432 \\
\hline 5 & $\mathrm{~m}$ & 41 & 1.10 & 0.24 & 2.04 & 0.65 & 0.376 & 0.188 & 0.495 & 0.248 \\
\hline 6 & $\mathrm{~m}$ & 51 & 1.20 & 0.35 & 1.50 & 0.44 & 0.486 & 0.189 & 0.646 & 0.264 \\
\hline 7 & $\mathrm{~m}$ & 41 & 1.03 & 0.28 & 0.87 & 0.25 & 0.465 & 0.187 & 0.591 & 0.263 \\
\hline 8 & $\mathrm{f}$ & 29 & 1.55 & 0.48 & 3.16 & 1.40 & 0.357 & 0.206 & 0.406 & 0.214 \\
\hline 9 & $\mathrm{~m}$ & 29 & 1.18 & 0.31 & 1.58 & 0.48 & 0.375 & 0.203 & 0.823 & 0.414 \\
\hline 10 & $\mathrm{f}$ & 27 & 1.18 & 0.37 & 1.95 & 0.71 & 0.380 & 0.179 & 0.541 & 0.258 \\
\hline Mean & & 34.50 & 1.27 & 0.35 & 2.01 & 0.69 & 0.407 & 0.204 & 0.607 & 0.282 \\
\hline SD & & 8.68 & 0.17 & 0.08 & 0.69 & 0.34 & 0.044 & 0.034 & 0.152 & 0.076 \\
\hline
\end{tabular}

$\mathrm{SD}^{*}$ denotes the intersubject standard deviation.

SD* bezeichnet die Standardabweichung zwischen den Individuen.

icant dispersion of the shear modulus values within the applied range of drive frequencies ( $25 \mathrm{~Hz}$ to $62.5 \mathrm{~Hz}$ ). Notably, MDEV-inversion used here sacrifices the dispersion-related information in order to stabilize the inversion and improve the certainty and precision of elastograms (i.e. $\left|G^{*}\right|$ - and $\varphi$-maps). This approach is fundamentally different from previous work in abdominal MRE where consistent shear modulus values were obtained by spatially averaging $G^{*}$ values within larger regions of the liver. At the moment, 'high-resolution' MRE cannot provide information about the frequency dependence of the measured mechanical parameters. However, further work is warranted to address this issue.
$\left|G^{*}\right|$ reflects the stiffness or softness of the tissue and is closely related to the shear wave speed measured by transient stimulation methods in ultrasound elastography. Our group mean value of $\left|G^{*}\right|=1.27 \pm 0.17 \mathrm{kPa}$ for the liver falls in the range of shear modulus values (1.1 to $1.8 \mathrm{kPa}$ ) measured by Fibroscan [36, 37] or ARFI $[37,38]$ ultrasound devices. The phase angle of the complex modulus $\varphi=\arctan \left[\operatorname{Im}\left(G^{*}\right) / \operatorname{Re}\left(G^{*}\right)\right]$ is a relatively unexplored parameter within the field of elastography. $\varphi$ is potentially sensitive to the geometry of the viscoelastic lattice [39]. According to the theory of viscoelastic scaling, $\varphi$ relates to the viscoelastic powerlaw constant (also referred to as the "loss tangent"), which can be directly linked to structure-relevant terms such as "fractal network dimension" or "network density" [39]. It is therefore not 
surprising that $\varphi$ is higher in the spleen than in the liver, given the fact that nearly $80 \%$ of the spleen is made up of red pulp, which consists of fibrils and connective tissue cells with a strongly cross-linked mechanical structure. However, the interpretation of $\varphi$ as a loss tangent implies the validity of the so-called springpot model [35], which we ignore in the recovery of $\left|G^{*}\right|$ using equation (6). MDEV inversion does not account for viscoelastic models but provides numerically stable solutions of two independent parameters related to the elastic and viscous behavior of the tissue. Similar to the storage modulus $\operatorname{Re}\left(G^{*}\right)$, we expect | $G^{*} \mid$ to be sensitive to fibrosis [13], whereas the significance of $\varphi$ as a diagnostic marker remains to be determined.

Our feasibility study in a group of ten healthy volunteers paves the road for further in-depth evaluation of mechanical imaging in the abdomen. Potential applications of high clinical relevance include the kidney [40], prostate [41, 42], and cervix [43, 44]. A further perspective of abdominal 3D-MMRE is the noninvasive assessment of tissue pressure by evaluating volumetric strain [19] or poroelastic parameters [45]. Applications in patients are warranted for the ultimate determination of the clinical value of the proposed respiratory gating method.

A limitation of the respiratory gating technique presented here is the need for patient cooperation. Patients who are not able to follow instructions have to be excluded from the examination. However, a similar problem arises in all MRI examinations involving actively controlled breathing. Therefore, our active gating approach in 3D-MMRE may be replaced in the future by passive respiratory gating such as navigator-based gating. At any rate, patient-activated 3D-MMRE, as proposed in this study, represents an advance toward the goal of solving the gating problem in abdominal MRE.

In summary, we have introduced a clinically feasible respiratory gating method for abdominal MRE providing highly resolved 3D wave fields at multiple harmonic vibration frequencies for the further improvement of mechanical parameter maps. Respiratory gating was achieved by a patient-activated pneumatic trigger. Using this novel method, we were able to acquire an unprecedented amount of information of in vivo abdominal shear waves in 10 transverse slices of $2.5 \mathrm{~mm}$ isotropic resolution with 3 field components, 7 vibration frequencies, and 8 time steps. We used this information to generate mechanical parameter maps of the magnitude and the phase of the complex shear modulus capable of displaying the heterogeneity of the in vivo shear modulus in abdominal organs. The significance of the enhanced MRE contrast for diagnostic applications such as the noninvasive staging of hepatic fibrosis or tumor detection remains to be determined.

\section{Appendix \\ $\nabla$}

\section{MDEV inversion}

Dual elasto-visco inversion relies on two independent solutions of different representations of the Helmholtz wave equation. We start with representing complex shear modulus $G^{*}$ by its magnitude $\left|G^{*}\right|$ and phase $\varphi$ :

$G^{*}=\left|G^{*}\right|(\cos \phi+i \sin \phi)$.

Corresponding to this representation, the Helmholtz equation can be written in terms of $\left|G^{*}\right|$ and $\varphi$ by

$\left|G^{*}\right|\left[\begin{array}{cc}\cos \phi & -\sin \phi \\ \sin \phi & \cos \phi\end{array}\right] \Delta \mathbf{x}_{\mathrm{mn}}=-\rho \omega_{\mathrm{n}}^{2} \mathbf{x}_{\mathrm{mn}}$ with $-\mathbf{x}_{\mathrm{mn}}=\left[\begin{array}{l}\mathrm{u}_{\mathrm{m}}^{\prime}\left(\omega_{\mathrm{n}}\right) \\ \mathrm{u}_{\mathrm{m}}^{\prime \prime}\left(\omega_{\mathrm{n}}\right)\end{array}\right]$, with $\Delta$ denoting the 3D-Laplacian operator and material density $\rho$ (assumed to be $1.1 \mathrm{~g} / \mathrm{cm}^{3}$ in the following [46]). $u_{m}^{\prime}\left(\omega_{n}\right)$ and $u_{m}^{\prime \prime}$ $\left(\omega_{n}\right)$ denote the real and imaginary part of $u_{m}^{*}\left(r, \omega_{n}\right)$, respectively, acquired at the vibration frequency $\omega_{n}$. As demonstrated in [18], equation (4) can be solved by

$\phi=\arccos \left(-\frac{\left.\sum_{m=1}^{3} \sum_{n=1}^{N}\left[\Delta u_{m}^{\prime}\left(\omega_{n}\right) u_{m}^{\prime}\left(\omega_{n}\right)+\Delta u_{m}^{\prime \prime}\left(\omega_{n}\right) u_{m}^{\prime \prime}\left(\omega_{n}\right)\right] \mid \Delta u_{m}^{*}\left(\omega_{n}\right) \| u_{m}^{*}\left(\omega_{n}\right)\right)}{\left.\sum_{m=1}^{3} \sum_{n=1}^{N}\left(\mid \Delta u_{m}^{\prime}\left(\omega_{n}\right)\right) \| u_{m}^{\prime}\left(\omega_{n}\right)\right)^{2}}\right)$.

A similar least-squares solution is obtained for $\left|G^{*}\right|$ by considering the magnitude of the Helmholtz equation $\left|G^{*}\right|\left|\Delta u_{m}^{*}\left(\mathbf{r}, \omega_{n}\right)\right|=\rho \omega_{n}^{2} \mid u_{m}^{*}$ $\left(\mathbf{r}, \omega_{n}\right) \mid$

$$
\left|G^{*}\right|=\rho \frac{\sum_{m=1}^{3} \sum_{n=1}^{N} \omega_{n}^{2}\left|\Delta u_{m}^{\prime}\left(\omega_{n}\right) \| u_{m}^{*}\left(\omega_{n}\right)\right|}{\sum_{m=1}^{3} \sum_{n=1}^{N}\left|\Delta u_{m}^{\prime}\left(\omega_{n}\right)\right|^{2}} .
$$

\section{Acknowledgement \\ $\nabla$}

This manuscript is dedicated to Professor Bernd Hamm for his 60th birthday.

\section{References}

1 Friedrich-Rust M, Ong MF, Martens $S$ et al. Performance of transient elastography for the staging of liver fibrosis: a meta-analysis. Gastroenterology 2008; 134: 960-974

2 Bonekamp S, Kamel I, Solga $S$ et al. Can imaging modalities diagnose and stage hepatic fibrosis and cirrhosis accurately? J Hepatol 2009; 50: $17-35$

3 Talwalkar JA, Kurtz DM, Schoenleber SJ et al. Ultrasound-based transient elastography for the detection of hepatic fibrosis: systematic review and meta-analysis. Clin Gastroenterol Hepatol 2007; 5: $1214-$ 1220

4 Castera $L$. Noninvasive methods to assess liver disease in patients with hepatitis B or C. Gastroenterology 2012; 142: 1293-1302 e1294

5 Vappou J. Magnetic resonance- and ultrasound imaging-based elasticity imaging methods: a review. Critical reviews in biomedical engineering $2012 ; 40: 121-134$

6 Friedrich-Rust M, Ong MF, Herrmann E et al. Real-time elastography for noninvasive assessment of liver fibrosis in chronic viral hepatitis. Am J Roentgenol Am J Roentgenol 2007; 188: 758 - 764

7 Ziol M, Handra-Luca A, Kettaneh A et al. Noninvasive assessment of liver fibrosis by measurement of stiffness in patients with chronic hepatitis C. Hepatology 2005; 41: $48-54$

8 Rouviere 0 , Yin M, Dresner MA et al. MR elastography of the liver: preliminary results. Radiology 2006; 240: 440-448

9 Palmeri ML, Wang MH, Dahl JJ et al. Quantifying hepatic shear modulus in vivo using acoustic radiation force. Ultrasound Med Biol 2008; 34: 546-558

10 Muthupillai R, Lomas DJ, Rossman PJ et al. Magnetic resonance elastography by direct visualization of propagating acoustic strain waves. Science 1995; 269 (5232): 1854 - 1857

11 Huwart L, Peeters F, Sinkus $R$ et al. Liver fibrosis: non-invasive assessment with MR elastography. NMR Biomed 2006; 19: 173-179

12 Yin M, Talwalkar JA, Glaser KJ et al. Assessment of hepatic fibrosis with magnetic resonance elastography. Clin Gastroenterol Hepatol 2007; 5 : 1207 - 1213 e 1202

13 Asbach P, Klatt D, Schlosser B et al. Viscoelasticity-based Staging of Hepatic Fibrosis with Multifrequency MR Elastography. Radiology 2010; 257: $80-86$

14 Hirsch S, Guo J, Reiter $R$ et al. MR Elastography of the Liver and the Spleen Using a Piezoelectric Driver, Single-Shot Wave-Field Acquisition, and Multifrequency Dual Parameter Reconstruction. Magnetic Resonance in Medicine 2013; DOI: 10.1002/jmri.24165

15 Doyley MM. Model-based elastography: a survey of approaches to the inverse elasticity problem. Phys Med Biol 2012; 57: R35-R73 
16 Klatt D, Asbach P, Rump J et al. In vivo determination of hepatic stiffness using steady-state free precession magnetic resonance elastography. Invest Radiol 2006; 41: 841-848

17 Dzyubak B, Glaser K, Yin M et al. Automated liver stiffness measurements with magnetic resonance elastography. J Magn Reson Imaging 2013; DOI: 10.1002/jmri.23980. [Epub ahead of print]

18 Papazoglou S, Hirsch S, Braun J et al. Multifrequency inversion in magnetic resonance elastography. Phys Med Biol 2012; 57: 2329-2346

19 Hirsch S, Klatt D, Freimann F et al. In vivo measurement of volumetric strain in the human brain induced by arterial pulsation and harmonic waves. Magn Reson Med 2012; doi: . DOI: 10.1002/mrm.24499. [Epub ahead of print]

20 Huwart L, Sempoux C, Vicaut E et al. Magnetic resonance elastography for the noninvasive staging of liver fibrosis. Gastroenterology 2008; 135: $32-40$

21 Garteiser P, Doblas S, Daire JL et al. MR elastography of liver tumours: value of viscoelastic properties for tumour characterisation. Eur Radiol 2012; 22: 2169-2177

22 Venkatesh SK, Yin M, Glockner JF et al. MR elastography of liver tumors: preliminary results. Am J Roentgenol Am J Roentgenol 2008; 190: $1534-1540$

23 Kamphues C, Klatt D, Bova R et al. Viscoelasticity-based magnetic resonance elastography for the assessment of liver fibrosis in hepatitis $C$ patients after liver transplantation. Fortschr Röntgenstr 2012; 184: $1013-1019$

24 Nedredal GI, Yin M, McKenzie T et al. Portal hypertension correlates with splenic stiffness as measured with MR elastography. J Magn Reson Imaging 2011; 34: 79-87

25 Uffmann K, Abicht C, Grote W et al. Design of an MR-Compatible piezoelectric actuator for MR elastography. Concept Magnetic Res 2002; 15 : $239-254$

26 Rump J, Klatt D, Braun J et al. Fractional encoding of harmonic motions in MR elastography. Magn Reson Med 2007; 57: 388 - 395

27 Papazoglou S, Xu C, Hamhaber U et al. Scatter-based magnetic resonance elastography. Phys Med Biol 2009; 54: 2229-2241

28 Hirsch S, Posnansky O, Papazoglou S et al. Measurement of vibration-induced volumetric strain in the human lung. Magn Reson Med 2012; doi: . DOI: 10.1002/mrm.24294. [Epub ahead of print]

29 Anderssen RS, Hegland M. For numerical differentiation, dimensionality can be a blessing! Math Comput 1999; 68 (227): 1121 - 1141

30 McLaughlin JR, Zhang N, Manduca A. Calculating tissue shear modulus and pressure by 2D Log-Elastographic methods. Inverse Probl 2010; 26

31 Sinkus $R$, Tanter $M$, Catheline $S$ et al. Imaging anisotropic and viscous properties of breast tissue by magnetic resonance-elastography. Magn Reson Med 2005; 53: 372-387

32 Huwart L, Sempoux C, Salameh $N$ et al. Liver fibrosis: noninvasive assessment with MR elastography versus aspartate aminotransferaseto-platelet ratio index. Radiology 2007; 245: 458-466
33 Talwalkar JA, Yin M, Venkatesh S et al. Feasibility of in vivo MR elastographic splenic stiffness measurements in the assessment of portal hypertension. Am J Roentgenol Am J Roentgenol 2009; 193: 122 - 127

34 Asbach P, Klatt D, Hamhaber $U$ et al. Assessment of liver viscoelasticity using multifrequency MR elastography. Magn Reson Med 2008; 60: $373-379$

35 Klatt D, Asbach P. Somasundaram R et al. Assessment of the Solid-Liquid Behavior of the Liver for the Diagnosis of Diffuse Disease Using Magnetic Resonance Elastography. Fortschr Röntgenstr 2008; 180: $1104-1109$

36 Bensamoun SF, Wang $L$, Robert $L$ et al. Measurement of liver stiffness with two imaging techniques: magnetic resonance elastography and ultrasound elastometry. J Magn Reson Imaging 2008; 28: 1287-1292

37 Crespo G, Fernandez-Varo G, Marino $Z$ et al. ARFI, FibroScan, ELF, and their combinations in the assessment of liver fibrosis: a prospective study. J Hepatol 2012; 57: $281-287$

38 Yoneda M, Suzuki K, Kato S et al. Nonalcoholic fatty liver disease: USbased acoustic radiation force impulse elastography. Radiology 2010; 256: $640-647$

39 Posnansky O, Guo J, Hirsch S et al. Fractal network dimension and viscoelastic powerlaw behavior: I. A modeling approach based on a coarse-graining procedure combined with shear oscillatory rheometry. Phys Med Biol 2012; 57: 4023-4040

40 Rouviere 0 , Souchon $R$, Pagnoux G et al. Magnetic resonance elastography of the kidneys: feasibility and reproducibility in young healthy adults. J Magn Reson Imaging 2011; 34: 880-886

41 Thormer G, Reiss-Zimmermann M, Otto J et al. Novel technique for MR elastography of the prostate using a modified standard endorectal coil as actuator. J Magn Reson Imaging 2013; DOI: 10.1002/jmri.23850. [Epub ahead of print]

42 Sahebjavaher RS, Baghani A, Honarvar $M$ et al. Transperineal prostate MR elastography: Initial in vivo results. Magn Reson Med 2013; doi: DOI: $10.1002 / \mathrm{mrm} .24268$. [Epub ahead of print]

43 Sun LT, Ning CP, Liu YJ et al. Is transvaginal elastography useful in preoperative diagnosis of cervical cancer? European journal of radiology 2012; 81: e888-e892

44 Thomas A, Kummel S, Gemeinhardt $O$ et al. Real-time sonoelastography of the cervix: tissue elasticity of the normal and abnormal cervix. Academic radiology 2007; 14: 193-200

45 Weaver JB, Pattison AJ, McGarry MD et al. Brain mechanical property measurement using MRE with intrinsic activation. Phys Med Biol 2012; $57: 7275-7287$

46 Lemke AJ, Brinkmann MJ, Schott T et al. Living Donor Right Liver Lobes: Preoperative CT Volumetric Measurement for Calculation of Intraoperative Weight and Volume. Radiology 2006 